\title{
The effect of hyperglycaemia on the macrophages in the cell culture
}

\author{
E.R. Megawati ${ }^{1}$, N. Meutia' ${ }^{1}$, L.D. Lubis ${ }^{2}$ \\ ${ }^{1}$ Department of Physiology, Faculty of Medicine, Universitas Sumatera Utara, Medan, Indonesia \\ ${ }^{2}$ Department of Histology, Faculty of Medicine, Universitas Sumatera Utara, Medan, Indonesia
}

[Received: 11 December 2020; Accepted: 27 January 2021; Early publication date: 23 February 2021]

Background: The availability of glucose in tissue has a role in macrophages polarisation into an inflammatory phenotype. The overnutrition condition such as hyperglycaemia induces macrophage infiltration especially the inflammatory macrophages. The aim of this study was to analyse the effect of hyperglycaemia condition on cytokines production by human monocytes-derived macrophages. Materials and methods: Monocyte cells obtained from peripheral blood mononuclear cells isolation from donors were incubated for 6 days in $37^{\circ} \mathrm{C}, 5 \% \mathrm{CO}_{2}$. On day 4, the stimulating factors such as lipopolysaccharide (LPS) and interferon gamma were added to activate monocytes into macrophages. Then, on day 6 , two doses of glucose; either normal or high doses along with low or high dose of LPS were given for $24 h$, followed by collecting the culture media and cells then stored at $-80^{\circ} \mathrm{C}$ until assayed.

Results: There was a significant difference in tumour necrosis factor alpha (TNF- $\alpha$ ) levels among groups, with highest level found in group with high-dose glucose plus high-dose LPS. However, the concentration of interleukin-6 (IL-6) among groups was not significantly different.

Conclusions: Macrophages treated with high-dose glucose plus high-dose LPS significantly increased production of TNF- $\alpha$, but not of IL-6. (Folia Morphol 2022; 81, 2: 387-393)

Key words: glucose, interleukin-6 (IL-6), lipopolysaccharides (LPS), macrophages, tumour necrosis factor alpha (TNF- $\alpha$ )

\section{INTRODUCTION}

Macrophage is one of innate immune cells that responds immediately to any invaders or cell debris. Macrophages have pathogen recognition receptors (PRRs) on its surface that can recognise pathogen-associated molecular patterns (PAMPs) or endogenous danger-associated molecular patterns (DAMPs) [7]. To bind to those molecules, macrophages are equipped with toll like receptors (TLR). NOD like-receptors (NLRs), the HIN-200 receptor family, and RIG-1-like receptor (RIRs) are also types of PRRs that can be found in macrophages which are located in the cytoplasm and act as stressor signals when pathogen is found in the cytoplasm [6].

Macrophages also have role to maintain tissue homeostasis [14], by polarising into two types of subpopulations, depending on the microenvironment they are exposed to [2]. The classical or proinflammatory macrophages are known as $\mathrm{M} 1$ and the alternative or anti-inflammatory macrophages as M2 [3].

Address for correspondence: Dr. E.R. Megawati, Jalan dr. T. Mansur No. 5 Kampus USU Medan 20155 Sumatera Utara, Indonesia, tel: +62618211045, fax: +62618216264, e-mail: eka3@usu.ac.id

This article is available in open access under Creative Common Attribution-Non-Commercial-No Derivatives 4.0 International (CC BY-NC-ND 4.0) license, allowing to download articles and share them with others as long as they credit the authors and the publisher, but without permission to change them in any way or use them commercially. 
The imbalance of the $\mathrm{M} 1 / \mathrm{M} 2$ ratio occurs in chronic inflammation condition such as insulin resistance, cardiovascular disease, obesity, tumour development, and autoimmune disease [3].

Macrophage infiltration also increases in the metabolically active tissue during overnutrition condition that leads to proinflammatory environment and is marked by the increase in tumour necrosis factor alpha (TNF- $\alpha$ ), interleukin (IL)- $1 \beta$, and inducible nitric oxide synthase (iNOs) secretion. In type 2 diabetes mellitus, macrophages involve in progressivity of the disease by altering metabolic pattern [5]. In obese patients, the M1 macrophages infiltrate the enlarged fat cells of adipose tissue and results in production of abundance of inflammatory cytokines such as TNF- $\alpha$ and IL-6. These contribute to the decrease in insulin sensitivity and the ability of glucose uptake in fat cells [6].

In the presence of interferon gamma (IFN- $\gamma$ ) and lipopolysaccharide (LPS), macrophages will rapidly activate their glycolytic pathway. Then, the abundance of glucose uptake and utilisation lead to the increases in IL- 6 and IL-1 $\beta$ secretion. Hence, the availability of glucose in a tissue may shift macrophages' polarization into an inflammatory phenotype [14]. In diabetes, the M1 macrophages persistently lead to diabetic neuropathy by over production of protease, cytokines and reactive oxygen species (ROS). These molecules induce oxidative stress and myelin degradation and neuronal damage. M1 macrophages may also change pancreatic cells functions and cause insulin resistance [1]. The M1 proinflammatory macrophages may produce, release and respond to cytokines and chemokines in its environment such as TNF- $\alpha$, IL-6, IL-1, and monocyte chemoattractant protein (MCP)-1. On the other hand, if the environments do not need the dominancy of proinflammatory macrophages, they adapt to the M2 macrophages. The anti-inflammatory macrophages $\mathrm{M} 2$ then allow the resolution of inflammation. The anti-inflammatory cytokines and the growth factors that are released by $\mathrm{M} 2$, such as the antagonist receptor IL-1, IL-10, and transforming growth factor beta, induce the decrease in the pro-inflammatory factors and then reduce the macrophages activation. The decrease in macrophages activation marked the end of acute inflammation. But, if the process is hampered, the resolution of inflammation cannot be achieved and chronic inflammation occurs [4].

The hyperglycaemic condition may activate monocyte and increase the expression of pro inflammatory macrophages (M1 phenotype), even if there is no tissue damage or infection [4]. Previous study showed that hyperglycaemic mice induced by streptozotocin had vast infiltration of macrophages and developed into a systemic proinflammatory state shown by the increase in IL-6, IL-1 $\beta$, TNF- $\alpha$, and IL-8 [10]. In an in vitro study, human monocytes and macrophages exposed to high levels of glucose also underwent transformation into inflammatory macrophages and the same situation apparently also can be observed in patients with hyperglycaemia [15].

The aim of the study was to analyse the effect of hyperglycaemia condition in the environment of the macrophage cell culture.

\section{MATERIALS AND METHODS}

\section{Subject recruitment}

The blood donors for this study were three females and one male with an average age of 20 years, body weight $64.5 \pm 8.44 \mathrm{~kg}$, height $161.5 \pm 1.66 \mathrm{~cm}$, and body mass index $25.20 \pm 3.30 \mathrm{~kg} / \mathrm{m}^{2}$. All participants were screened for eligible criteria, such as not having any infection and metabolic diseases in 1 month before and also signed the informed consent after being explained about the study procedures. All procedures of this study were approved by the Ethical Committee of Human Studies, Faculty of Medicine and Adam Malik General Hospital, Medan. This study was conducted in the Research Laboratory of Faculty of Medicine, Universitas Sumatera Utara, Medan, Indonesia between July and October 2019.

\section{Isolation of PBMCs}

Fifty millilitres of blood was withdrawn from a vein and collected in EDTA-containing Vacutainer tubes. The peripheral blood mononuclear cells (PBMCs) of buffy coat were collected by gradient centrifugation using Lymphoprep ${ }^{\mathrm{TM}}$ (Stemcell, Axis-Shield, Dundee, UK), according to the manufacturer's instruction.

\section{Isolation of monocytes}

Monocytes were isolated from PBMCs by using EasySep $^{\mathrm{TM}}$ negative monocyte isolation kit (Stemcell $^{\mathrm{TM}}$ Technologies, Vancouver, Canada). Briefly, the PBMCs were treated with antibody and platelet removal before selected with magnet beads and incubate in an EasySep Magnet (Stemcell ${ }^{\mathrm{TM}}$ Technologies, Vancouver, Canada) according to the manufacture's procedures. 


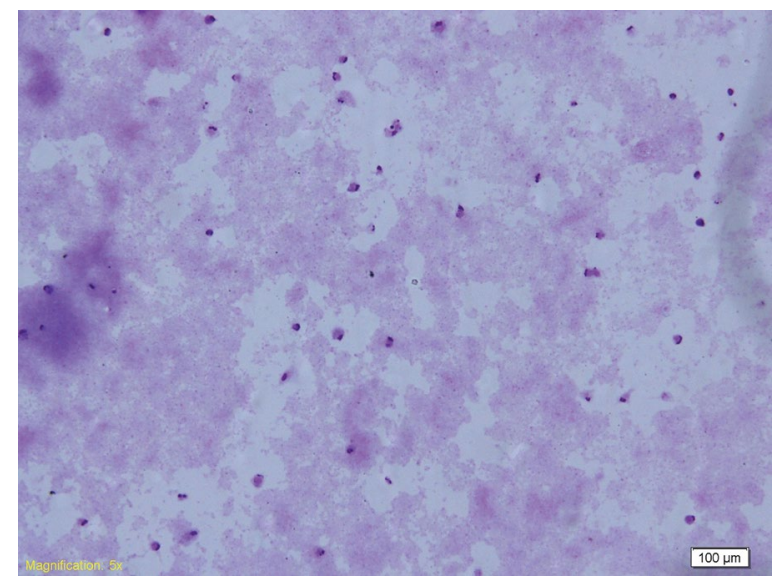

Figure 1. Normal glucose $10 \times 10$.

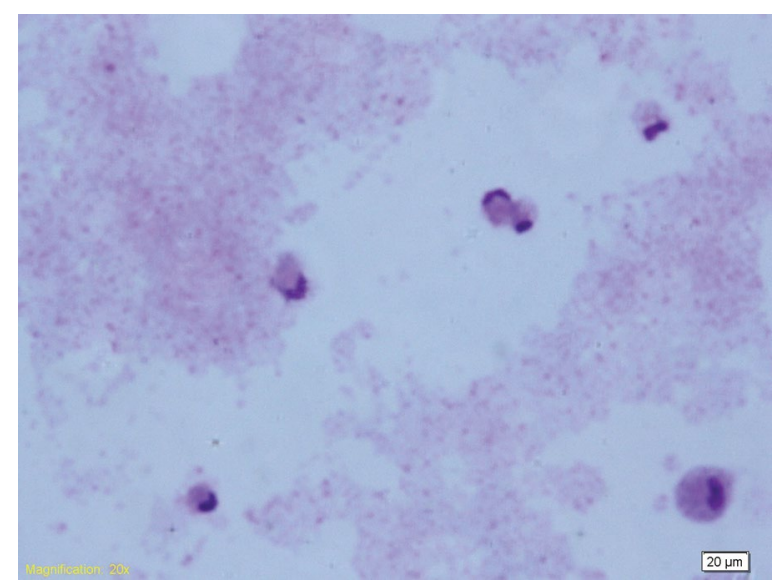

Figure 2. Normal glucose $10 \times 40$.

\section{Culture of monocytes}

Monocytes were cultured in RPMI 1640 (Gibco, Waltham, MA, USA) supplemented with $10 \%$ foetal bovine serum (FBS; Sigma, St. Louis, MO, USA), 1\% penicillin-streptomycin (Gibco, Waltham, MA, USA) and $1 \%$ L-glutamine (Gibco, Waltham, MA, USA) at $37^{\circ} \mathrm{C}$ in a $5 \% \mathrm{CO}_{2}$ humidified incubator. Recombinant human M-CSF/CSF1 (ABclonal Technology, Woburn, MA, USA) at the concentration of $50 \mathrm{ng} / \mathrm{mL}$ was used to direct macrophage differentiation. Cells were incubated for 6 day in $37^{\circ} \mathrm{C}, 5 \% \mathrm{CO}_{2}$. The monocytes seeded in 24 wells culture plates (Iwaki tissue culture treated polystyrene microplate, Asahi Glass Co., Japan). On day 4, fresh media were added with lipopolysaccharide (LPS; $10 \mathrm{ng} / \mathrm{mL}$ ) from E. coli (O26:B6) (Sigma, St. Louis, MO, USA) and IFN- $\gamma$ $(50 \mathrm{ng} / \mathrm{mL})$ (Stemcell $^{\mathrm{TM}}$ Technologies, Vancouver,

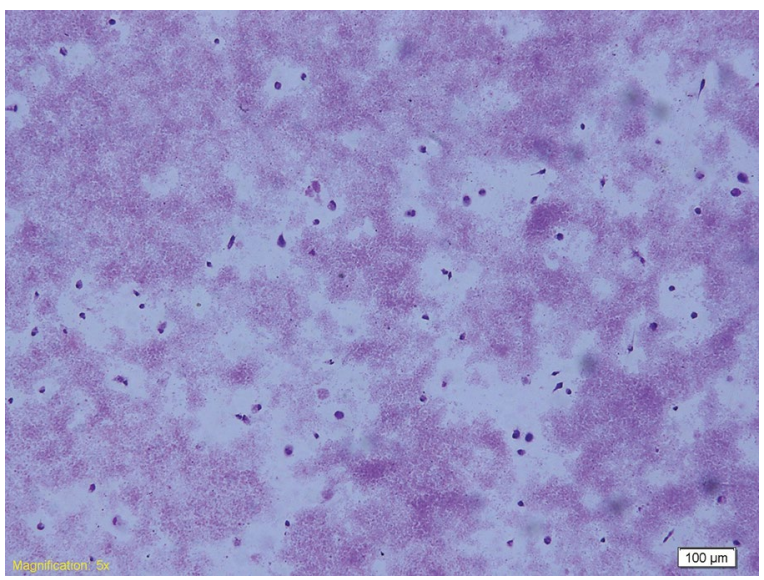

Figure 3. High glucose $10 \times 10$.

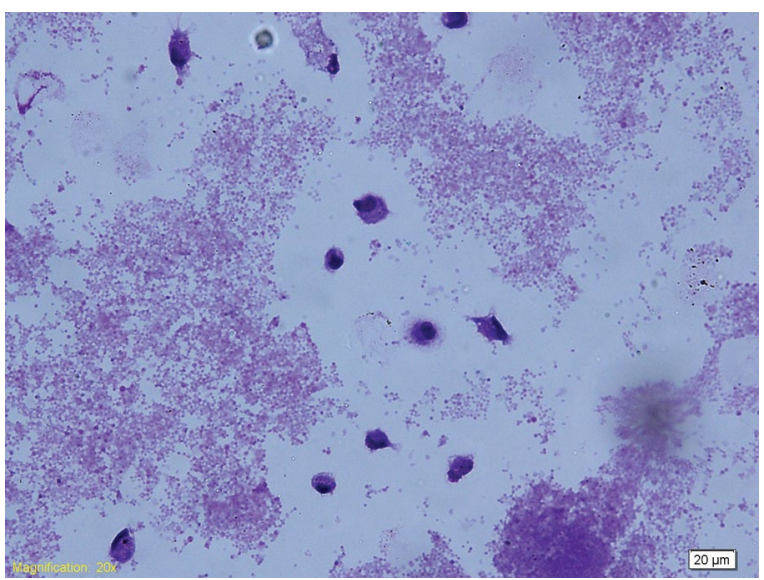

Figure 4. High glucose $10 \times 40$.

Canada) to differentiate macrophage into M1 macrophages. On day 6 , all media were removed and replaced with serum-free complete media (SF-CM).

\section{Glucose stimulation}

The macrophages were divided into five groups; two were supplemented with two doses of glucose (Sigma, St. Louis, MO, USA) (7 mM as normal dose [ND-Glu] and $35 \mathrm{mM}$ as high dose [HD-Glu]). This concentration is based on the diabetes diagnosis by World Health Organization [16] and doubling the dosage will resemble the diabetes state in patients [4]. The other three groups besides having the two doses of glucose were also supplemented with LPS (low dose $100 \mathrm{ng} / \mathrm{mL}$ [LD-LPS] and high doses $2 \mu \mathrm{g} / \mathrm{mL}$ [HD-LPS]). The control group was treated with SF-CM. The treatments were given for $24 \mathrm{~h}$ after day 6 . Then 
the culture media and cells were harvested and frozen at $-80^{\circ} \mathrm{C}$ until assayed.

\section{Giemsa staining}

The coverslips that previously put inside the wells were discarded and left dry in room temperature. Then fixed with $98 \%$ methanol for $1 \mathrm{~min}$ and bathed with Giemsa (diluted with distilled water, 1:1 vol/vol) for $5 \mathrm{~min}$. In the final step, the coverslips were washed with distilled water and left to dry then mounted onto slides. The Giemsa staining of macrophages stimulated with normal dose of glucose is seen in Figures 1 and 2 and in high dose of glucose in Figures 3 and 4, with $10 \times 10$ and $10 \times 40$ magnification.

\section{Analysis of cytokines production}

Human Pico Kine ELISA kits (My BioSource Inc., San Diego, CA, USA) were used to analyse the concentration of TNF- $\alpha$ and IL- 6 according to manufacturer's protocols. The assays were performed in duplicates. The intra-assay coefficient of variation was $<11 \%$.

\section{Statistical analysis}

All data are presented as mean \pm standard deviation. All data were normally distributed after analysed using Shapiro-Wilk test, then were analysed with one way ANOVA and followed by Bonferroni post hoc test. The $p$ value $<0.05$ was considered statistically significant. All data were statistically analysed using SPSS software version 17.0.

\section{RESULTS}

\section{The production of TNF- $\alpha$}

Test of homogeneity of variances with Levene's test showed that there were significant differences in variance with $p$-value $=0.001$, then one way ANOVA Welch test showed that the data were significantly different among groups with $p$ value $=0.004(p<0.05)$. The Robust Test of Equality of Means showed the $p$ value $=0.29$ ( $p>0.05)$ (equals variances assumed), so the analysis was continued with the Bonferroni post hoc test to find which groups were significantly different.

As can be seen in Table 1, the levels of TNF- $\alpha$ were significantly different among groups with $p=0.004$, the group treated with normal dose of glucose had lowest TNF- $\alpha$ concentration (40.67 $\pm 9.94 \mathrm{pg} / \mathrm{mL}$ ), while in the group treated with high dose of glucose plus high dose of LPS had highest TNF- $\alpha$ concentration $(2150.22 \pm 1379.77 \mathrm{pg} / \mathrm{mL})$.
This study showed that macrophages treated with normal dose or high dose of glucose and/or stimulated with low dose or high dose of LPS had higher concentration of TNF- $\alpha$ compared to the treated macrophages. Then, after being analysed with the Bonferroni post hoc test, it was shown that there are significant differences in TNF- $\alpha$ concentration within groups; Control vs. HD-Glu+HD-LPS (mean differences $[M D]=-2107.34 \mathrm{pg} / \mathrm{mL} ; \mathrm{p}=0.015)$, ND-Glu vs. HD-Glu +HD-LPS (MD $=-1943.53 \mathrm{pg} / \mathrm{mL}$; $\mathrm{p}=0.009$ ), and HD-Glu vs. HD-Glu+HD-LPS (MD $=-2095.69 \mathrm{pg} / \mathrm{mL}, \mathrm{p}=0.002$ ). It means that high dose of glucose plus high dose of LPS resulted in an increase in TNF- $\alpha$ production (Table 2).

\section{The production of IL- 6}

This study showed that the lowest concentration of IL-6 were found in the group treated with a high dose of glucose $(43.37 \pm 16.29 \mathrm{pg} / \mathrm{mL})$, whereas the highest concentration was found in the group treated with a high dose of glucose plus a high dose of LPS $(59.66 \pm 16.72 \mathrm{pg} / \mathrm{mL})$. But after being analysed with one way ANOVA test, the concentrations of IL- 6 were not significantly different ( $p=0.742$; Table 3 ).

\section{The macrophages staining}

This study also performed the Giemsa staining of macrophages with normal-dose and high-dose glucose. The figures are shown with $10 \times 10$ and $10 \times 40$ magnification (Figs. 1-4).

\section{DISCUSSION}

We observed that the highest level of TNF- $\alpha$ was found in the HD-Glu+HD-LPS group, and the lowest was found in the control group ( $p=0.004)$. This finding is similar to previous study that compared the production of proinflammatory cytokines in macrophages that stimulated with LPS to the non-stimulated macrophages. The previous study showed that the production of TNF- $\alpha$, MCP-1, IL- 6 and IL- $1 \beta$ was significantly higher in stimulated macrophages compared to control [1]. Previous study also showed that THP-1 macrophage cultured in high glucose concentration (15 mM) with or without LPS stimulation displayed higher concentration of TNF- $\alpha$ compared to normal glucose (5 mM) or osmotic control group [4]. Furthermore, activated macrophages that were stimulated with hyperglycaemia and hypoxia condition were shown to upregulate TNF- $\alpha$, IL-6 and IL-1 gene expression [9]. Another study on diabetic rats induced 
Table 1. The TNF- $\alpha$ level among groups

\begin{tabular}{lcc}
\hline Groups & TNF- $\alpha$ [pg/mL] & P value \\
\hline Control & $42.88 \pm 22.85$ & $0.004^{*}$ \\
ND-Glu & $40.67 \pm 9.94$ & \\
HD-Glu & $54.43 \pm 26.93$ & \\
ND-Glu+HD-LPS & $915.09 \pm 804.02$ & \\
HD-Glu+LD-LPS & $979.49 \pm 8226.84$ & \\
HD-Glu+HD-LPS & $2150.22 \pm 1379.77$ & \\
\hline
\end{tabular}

Values are mean \pm standard deviation $(n=4)$. TNF- $\alpha$ - tumour necrosis factor alpha; Control — serum-free media only; ND-Glu — normal-dose glucose, $7 \mathrm{mM}$; HD-Glu -

high-dose glucose, $35 \mathrm{mM}$; LD-LPS — low-dose lipopolysaccharides, $100 \mathrm{ng} / \mathrm{mL}$; HD-LPS - high-dose lipopolysaccharides, $2 \mu \mathrm{g} / \mathrm{mL}^{*} \mathrm{p} p<0.05$ (the one way ANOVA test)
Table 3. The IL-6 level among groups

\begin{tabular}{lcc}
\hline Groups & IL-6 [pg/mL] & P value \\
\hline Control & $55.16 \pm 23.19$ & 0.742 \\
ND-Glu & $49.52 \pm 29.28$ & \\
HD-Glu & $43.37 \pm 16.29$ & \\
ND-Glu+HD-LPS & $53.06 \pm 20.45$ & \\
HD-Glu+LD-LPS & $52.08 \pm 23.85$ & \\
HD-Glu+HD-LPS & $59.66 \pm 16.72$ & \\
\hline
\end{tabular}

Values are mean \pm standard deviation $(n=4)$. IL-6 - interleukin-6; Control - serum -free media only; ND-Glu — normal-dose glucose, $7 \mathrm{mM}$; HD-Glu — high-dose glucose $35 \mathrm{mM}$; LD-LPS — low-dose lipopolysaccharides, $100 \mathrm{ng} / \mathrm{mL}$; HD-LPS — high-dose lipopolysaccharides, $2 \mu \mathrm{g} / \mathrm{mL}^{*}{ }^{*} \mathrm{p}<0.05$ (the one way ANOVA test)

Table 2. The post hoc test of tumour necrosis factor alpha level among groups

\begin{tabular}{lcccc}
\hline Groups & Mean differences & \multicolumn{2}{c}{$95 \%$ confidence interval } & P value \\
\cline { 2 - 4 } & & Lower bound & Upper bound & 1.00 \\
\cline { 2 - 3 } Control vs. ND-Glu & -163.81 & 1852.16 & 1524.53 & 1.00 \\
Control vs. HD-Glu & -11.65 & 1661.18 & 1637.88 & 1.00 \\
Control vs. HD-Glu+LD-LPS & -936.61 & 2841.33 & 968.09 & 1.00 \\
Control vs. ND-Glu+HD-LPS & -872.21 & 2776.92 & 1032.50 & $0.015^{*}$ \\
Control vs. HD-Glu+HD-LPS & -2107.34 & 4012.05 & 202.63 & 1.00 \\
ND-Glu vs. HD-Glu & -152.16 & 1241.94 & 1546.27 & 1.00 \\
ND-Glu vs. HD-Glu+LD-LPS & -772.80 & 2461.15 & 915.54 & 1.00 \\
ND-Glu vs. ND-Glu+HD-LPS & -708.40 & 2396.74 & 979.95 & $0.009^{*}$ \\
ND-Glu vs. HD-Glu+HD-LPS & -1943.53 & 3631.87 & 255.18 & 1.00 \\
HD-Glu vs. HD-Glu+LD-LPS & -924.96 & 2574.49 & 724.56 & 1.00 \\
HD-Glu vs. ND-Glu+LD-LPS & -860.56 & 2510.09 & 788.97 & $0.002^{*}$ \\
HD-Glu vs. HD-Glu+HD-LPS & -2095.69 & 3745.22 & 446.16 & 1.00 \\
HD-Glu+LD-LPS vs. ND-Glu+HD-LPS & 64.41 & 1840.30 & 1968.12 & 1.00 \\
HD-Glu+LD-LPS vs. HD-Glu+HD-LPS & -1170.72 & 3075.43 & 733.99 & 1.00 \\
ND-Glu+HD-LPS vs. HD-Glu+HD-LPS & -1235.13 & 3139.84 & 669.58 & \\
\hline
\end{tabular}

Control — serum-free media only; ND-Glu — normal-dose glucose, 7 mM; HD-Glu — high-dose glucose, 35 mM; LD-LPS — low-dose lipopolysaccharides, $100 \mathrm{ng} / \mathrm{mL}$; HD-LPS — high-dose lipopolysaccharides, $2 \mu \mathrm{g} / \mathrm{mL}^{*}{ }^{*} \mathrm{p}<0.05$ (Bonferroni post hoc test)

by streptozotocin also shown an increase in TNF- $\alpha$ and overexpression of inflammatory genes, such as TNF- $\alpha$, COX-2 and iNOS [11].

Differences in TNF- $\alpha$ concentration was observed among groups; Control vs. HD-Glu+HD-LPS, ND-Glu vs. HD-Glu+HD-LPS, and HD-Glu vs. HD-Glu+HD-LPS with $p$ value of $0.015,0.009$, and 0.002 ( $p<0.05)$, respectively. The macrophages with high-dose glucose plus high-dose LPS produced significantly higher levels of TNF- $\alpha$ compared to the serum free medium (control), or to the macrophages with normal or high-dose glucose.

The TNF- $\alpha$, IFN- $\gamma$, or the recognition of LPS are the Th1 (T helper 1) cytokines that can trigger the polar- ization of proinflammatory M1 macrophages. The activity of these M1 macrophages will then increase the expression of IL-12, IL-23, and the production of TNF, IL- $1 \beta$ and IL- 6 , and also ROS via activation of nicotinamide adenine dinucleotide phosphate (NADPH) oxidase system. The function of this action is to remove pathogens during infection that results in ROS-mediated tissue damage and declined wound healing [8]. It also has been known that the circulating LPSs bind with LPS binding protein (LBP) and are caught by CD14 surface scavenging receptor. This signal activates TLR that initiates the phosphorylation cascade mediated by different kinase enzymes. The result of this signal is releasing transcription 
factor - nuclear factor kappa B from its inhibitor $\mid \kappa \mathrm{B}-$ and translocating it to the nucleus. The effect of this cascade is increasing the expression of inflammatory genes such as TNF, antibiotic peptides and NADPH oxidase that produce the reactive oxygen intermediate [13].

We observed that the highest level of IL- 6 was found in HD-Glu+HD-LPS and the lowest was found in HD-Glu, but the differences were not significant $(p>0.05)$. This study showed that the IL-6 production was not changed by glucose and/or LPS stimulation. This result is in contrary to previous study that analysed the differences in cytokine production among type 2 diabetes patients and diabetic peripheral neuropathy patients; the macrophages from patients with type 2 diabetes produced significantly higher level of IL-6 when compared to control and diabetic peripheral neuropathy patients [1]. Another study also showed that THP-1 macrophages produced higher level of IL- 6 when stimulated with high glucose and LPS, compared to control group. The concentration of normal glucose in that study was $5 \mathrm{mM}$ and the high glucose was $15 \mathrm{mM}$ [4]. It means that M1 macrophages are more metabolically active in hyperglycaemic conditions, and promote obesity associated insulin resistance [12]. The difference between this finding and those studies is the dose being used. In addition, the dramatically increased IL- 6 gene expression was seen after $17 \mathrm{~h}$ of activation and stimulation with hyperglycaemic along with hypoxia [9], whereas in this study the concentration of IL-6 was analysed after $24 \mathrm{~h}$ of stimulation. Similar results were also found in the previous study of monocyte-derived macrophages exposed to high glucose $(15 \mathrm{mM})$ that showed no significant differences in TNF- $\alpha$ production when compared to the normal glucose $(5 \mathrm{mM})$ or hyperosmotic (glucose free) conditions [15].

The limitation of this study is we did not perform the immunophenotypic assessment that is useful to identify and count the percentage of cells. Anyhow, we identify the cells using the cytomorphology and the number of cells with improved Neubauer chamber.

\section{CONCLUSIONS}

Finally, this study concluded that macrophages which were stimulated with a high dose of glucose plus a high dose of LPS significantly increased the TNF- $\alpha$ production. On the other hand, different dosages of glucose and LPS stimulation to the macrophages cell cultures did not change the production of IL-6.

\section{Acknowledgements}

This study was supported by Ministry of Research and Technology and Higher Education Republic of Indonesia under the research grants of TALENTA Universitas Sumatera Utara Year 2019 with contract number 4167/UN5.1.R/PPM/2017, on April 1t, 2019.

\section{Conflict of interest: None declared}

\section{REFERENCES}

1. Alvarado-Vázquez PA, Grosick RL, Moracho-Vilrriales C, et al. Cytokine production capabilities of human primary monocyte-derived macrophages from patients with diabetes mellitus type 2 with and without diabetic peripheral neuropathy. J Pain Res. 2019; 12: 69-81, doi: 10.2147/JPR. S186372, indexed in Pubmed: 30588081.

2. Arnold CE, Gordon P, Barker RN, et al. The activation status of human macrophages presenting antigen determines the efficiency of Th17 responses. Immunobiology. 2015; 220(1): 10-19, doi: 10.1016/j.imbio.2014.09.022, indexed in Pubmed: 25454489.

3. Figueroa LA, Abarca-Vargas R, García Alanis C, et al. Comparison between Peritoneal Macrophage Activation by Extract and LPS and/or Interleukins. Biomed Res Int. 2017; 2017: 4602952, doi: 10.1155/2017/4602952, indexed in Pubmed: 29279849.

4. Grosick R, Alvarado-Vazquez PA, Messersmith AR, et al. High glucose induces a priming effect in macrophages and exacerbates the production of pro-inflammatory cytokines after a challenge. J Pain Res. 2018; 11: 1769-1778, doi: 10.2147/JPR.S164493, indexed in Pubmed: 30237731.

5. Hameed I, Masoodi SR, Mir SA, et al. Type 2 diabetes mellitus: From a metabolic disorder to an inflammatory condition. World J Diabetes. 2015; 6(4): 598-612, doi: 10.4239/wjd.v6.i4.598, indexed in Pubmed: 25987957.

6. Hirayama $D$, lida $T$, Nakase $H$. The phagocytic function of macrophage-enforcing innate immunity and tissue homeostasis. Int J Mol Sci. 2017; 19(1), doi: 10.3390/ ijms19010092, indexed in Pubmed: 29286292.

7. Kelly B, O'Neill LAJ. Metabolic reprogramming in macrophages and dendritic cells in innate immunity. Cell Res. 2015; 25(7): 771-784, doi: 10.1038/cr.2015.68, indexed in Pubmed: 26045163.

8. Momtazi-Borojeni AA, Abdollahi E, Nikfar B, et al. Curcumin as a potential modulator of $\mathrm{M} 1$ and $\mathrm{M} 2$ macrophages: new insights in atherosclerosis therapy. Heart Fail Rev. 2019; 24(3): 399-409, doi: 10.1007/s10741-018-09764-z, indexed in Pubmed: 30673930.

9. Morey M, O'Gaora P, Pandit A, et al. Hyperglycemia acts in synergy with hypoxia to maintain the pro-inflammatory phenotype of macrophages. PLoS One. 2019; 14(8): e0220577, doi: 10.1371/journal.pone.0220577, indexed in Pubmed: 31415598.

10. Niu S, Bian Z, Tremblay A, et al. Broad infiltration of macrophages leads to a proinflammatory state in streptozotocin-induced hyperglycemic mice. J Immunol. 2016; 197(8): 3293-3301, doi: 10.4049/jimmunol.1502494, indexed in Pubmed: 27619992. 
11. Pan Y, Wang Yi, Cai Lu, et al. Inhibition of high glucose-induced inflammatory response and macrophage infiltration by a novel curcumin derivative prevents renal injury in diabetic rats. Br J Pharmacol. 2012; 166(3): 1169-1182, doi: 10.1111/j.14765381.2012.01854.x, indexed in Pubmed: 22242942.

12. Riemann $S$, Klüter $H$, Gratchev $A$, et al. Hyperglycemia induces mixed M1/M2 cytokine profile in primary human monocyte-derived macrophages. Immunobiology. 2017; 222(10): 952-959, doi: 10.1016/j.imbio.2016.07.006, indexed in Pubmed: 27492721.

13. Roitt IM, Delves PJ. Roitts's Essential Immunology. 10th ed. Blackwell Science 2001: 1-494.
14. Thapa B, Lee K. Metabolic influence on macrophage polarization and pathogenesis. BMB Reports. 2019; 52(6): 360-372, doi: 10.5483/bmbrep.2019.52.6.140.

15. Torres-Castro I, Arroyo-Camarena ÚD, Martínez-Reyes CP, et al. Human monocytes and macrophages undergo M1-type inflammatory polarization in response to high levels of glucose. Immunol Lett. 2016; 176: 81-89, doi: 10.1016/j.imlet.2016.06.001, indexed in Pubmed: 27269375.

16. World Health Organization (WHO). Global report on diabetes. 2016. https://www.who.int/publications/i/ item/9789241565257. 